\title{
Design and Synthesis of Zwitterionic Metal-Organic Frameworks
}

\author{
$\underline{\text { Jenna Brockett }^{1}}$, Shefa Alomari², Dr. Mario Wriedt ${ }^{2}$ \\ ${ }^{1}$ Department of Chemical and Biomolecular Engineering, Clarkson University \\ ${ }^{2}$ Department of Chemistry and Biomolecular Science, Clarkson University \\ brockej@clarkson.edu
}

Metal-organic frameworks (MOFs) have emerged as a new type of porous material. Their crystalline structures are composed of metal clusters connected by polytypic organic linkers. The characteristics of the metal and organic ligand blocks are important for controlling the resulting topologies, pore environments, and functionalities and hence affect the characteristics of the MOFs to capture specific guest molecules. As a new means to introduce charged-polarized porosity into MOFs we explore zwitterionic (ZW) ligands as MOF building blocks. These ligands are composed of both cationic and anionic functional groups resulting in an electrostatic field gradient on their molecular surfaces. Incorporating them into MOFs leads to polarization effects on guest molecules, resulting in enhanced adsorption enthalpies and multi-stimulusresponsive properties. N-substituted pyridines, namely pyridinium and their derivatives are among the most famous zwitterions; however, the design and synthesis of new pyridinium-based ligands are challenging. In this research, the synthesis of new rigid zwitterionic ligands through multi-non-standard organic protocols is explored. This poster presentation will address the crystal structures and physical properties of new ZW MOFs. It will be shown that these systematic investigations will pave the way to develop new functional materials with improved adsorption properties.

Acknowledgements: Wriedt Laboratory, Collegiate Science and Technology Entry Program, Community of Underrepresented Professional Opportunities, Arthur O. Eve Higher Education Opportunity Program, and the Clarkson University Honors Program for their support.

Mentors: Dr. Mario Wriedt and Shefa Alomari, Department of Chemistry, Clarkson University.

Keywords: Metal-organic framework, Zwitterionic ligand, Porous material, Pyridinium derivatives 